Conclusion This study showed substantial competing interests amongst scientists commenting on the use of antivirals and/or vaccines in H1N1 influenza during the period the UK government was deciding its pharmaceutical policy. Since commentaries in the media provide an alternative route for external pressure on health policy decisions, scientists should declare any potential competing interests for media interviews as for journal articles.

\section{OP79 YOUTH EXPOSURE TO TELEVISION ALCOHOL ADVERTISING IN THE UK}

doi:10.1136/jech-2012-201753.079

${ }^{1} \mathrm{~S}$ Patil, ${ }^{2} \mathrm{M}$ N Elliott, 'E M Winpenny, 'C Rohr, 'E Nolte. 'N/A, RAND Europe, Cambridge, UK; ${ }^{2} N / A$, RAND Corporation, Santa Moica, CA, USA

Background Increasing evidence suggests that exposure to alcohol marketing increases the likelihood that adolescents will start to drink alcohol and that among those who already consume alcohol exposure to alcohol advertising is likely to increase its use. Existing research in Europe has tended to focus on the content of alcohol marketing and advertising but our understanding of the actual levels of exposure remains inadequate. This study aims to contribute to filling this gap by analysing youth exposure to television alcohol advertising in the UK.

Methods We obtained data on viewership and on alcohol advertising volume for the top ten television channels with the highest number of viewers in the UK by age (4-9, 10-15, 16-24, 25 plus) for December 2010 to May 2011. Data were analysed descriptively to characterise youth viewership by channel, month and time of the day ('daypart') and alcohol advertising exposure. We then used a negative binomial regression model to measure incidental youth exposure to alcohol advertising relative to adults over 25 years of age. We applied sensitivity analyses to test robustness of the model. Results Viewership and alcohol advertising volume varied substantially across the 10 channels, months and dayparts. Children and adolescents (10-15 years) constituted about $4 \%$ of the total audience and young people (16-24 years) 8\%, yet their exposure to alcohol commercials was $5 \%$ and $50 \%$, respectively. Relative to adults 25 years of age and older, the regression analysis found advert intensity was higher where children and adolescents constituted a greater proportion of the viewership (incident rate ratio (IRR) 1.09, $95 \%$ CI 1.02, 1.17, $p=0.013$ ). This relationship was particularly strong for commercials of beer and cider (IRR $=1.14,95 \%$ CI 1.06 , $1.23, \mathrm{p}=0.000)$, super market brands (IRR $=1.16,95 \%$ CI 1.07, 1.28, $\mathrm{p}=0.000$ ), and ready-mix drinks (IRR $=1.51,95 \%$ CI 1.27, 1.78, $p=0.000$ ). In contrast, although the IRR for those aged 16 to 24 years also differed significantly from that observed for older adults, the difference was small $(1.02,95 \%$ CI 1.01, 1.04, $p=0.003)$. There were no statistically significant associations for wine and spirit advert incidence and viewership for any of the younger population groups.

Conclusion Our findings suggest that young people in the UK have a disproportionately higher exposure to television alcohol (except wine and spirits) advertising than would be expected from general viewership patterns. Alcohol advertising practices should be modified to limit exposure of underage viewers.

\section{OP80 MINIMUM UNIT PRICING FOR ALCOHOL: A DOCUMENT ANALYSIS OF EVIDENCE SUBMISSIONS TO THE SCOTTISH PARLIAMENT}

doi:10.1136/jech-2012-201753.080

'S V Katikireddi, ${ }^{2}$ S Hilton, 'L Bond. 'Evaluating the Health Effects of Social Interventions programme, MRC/CSO Social and Public Health Sciences Unit, Glasgow, UK; ${ }^{2}$ Understandings and Uses of Public Health Research, MRC/CSO Social and Public Health Sciences Unit, Glasgow, UK
Background Minimum unit pricing (MUP) of alcohol is a novel policy intervention aimed at increasing the price of the cheapest forms of alcohol to reduce alcohol consumption and associated harms. In Scotland, alcohol-related harms have increased exponentially during the past few decades and Scotland currently experiences the greatest level of alcohol-related mortality within the UK. Scotland would be the first country in the world to introduce the measure and therefore both advocates and critics have seized the opportunity to put forward the different arguments in the case of MUP. This study examines the evidence submitted to the Scottish Parliament with the aim of examining how arguments for and against MUP have been framed and to consider what forms of evidence have been drawn upon by different policy stakeholders to debate MUP.

Methods The Scottish Parliament received evidence submissions from a wide variety of stakeholders in its first consideration of MUP as part of the Stage 1 scrutiny process for the Alcohol (etc.) Bill 2010. Sixty-five documents submitted by 47 different stakeholders (including politicians, health professionals, industry representatives, supermarkets and researchers) considered within meetings of the Select Committee were analysed. Data management was assisted using Nvivo 9, framework matrices, and thematically analysed using constant comparative methods.

Results Arguments around alcohol policy have been actively constructed as a health issue by advocates for MUP in comparison to critics who highlight economic, ideological, and social aspects. Constructions of alcohol overconsumption as a population health issue, rather than an individual-level health problem, were contested. Alcohol epidemiology was drawn upon by advocates (to support a whole population approach) but also by critics (to argue alcohol harms are reducing). Issues raised in opposition include important potential secondary impacts such as exacerbating inequalities, increasing cross-border and illicit trade and perverse incentives (such as increases in profitability driving retailers to encourage increased sales).

Conclusion Framing of alcohol policy as a health (rather than, for example, trade or justice) issue that requires a whole population approach has been important in creating conditions amenable to MUP being seriously considered by policymakers. The initial framing of an issue in different ways can lead to similar sources of evidence, such as routine epidemiological data, being used to suggest different actions are appropriate. Public health researchers and practitioners should be aware of the active construction process occurring in policy definition and adapt research and advocacy accordingly.

\section{Public Health Interventions: Diet}

\section{OP81 ESTIMATING THE POTENTIAL OF POPULATION LEVEL CHANGES IN CHOLESTEROL AND BLOOD PRESSURE FOR REDUCING UK CORONARY HEART DISEASE MORTALITY RATES: A NOVEL MODELLING APPROACH}

doi:10.1136/jech-2012-201753.081

${ }^{1} \mathrm{M}$ O'Flaherty, ${ }^{2} \mathrm{P}$ Couch, ${ }^{2} \mathrm{M}$ Sperrin, ${ }^{2} \mathrm{~N}$ Green, ${ }^{2} \mathrm{~J}$ Ainsworth, ${ }^{3} \mathrm{M}$ Huffmann, ${ }^{3} \mathrm{D}$ LloydJones, ${ }^{2}$ Buchan, ${ }^{1} \mathrm{~S}$ Capewell. 'Deaprtment of Public Health and Policy, University of Liverpool, Liverpool, UK; ${ }^{2}$ Northwest Institute for Biohealth Informatics, University of Manchester,Manchester UK; ${ }^{3}$ Department of Preventive Medicine, Northwestern University, Chicago, USA

Background Coronary heart disease (CHD) mortality is declining. However, population ageing and increasing obesity and diabetes might reverse this trend. Strategies to reduce salt and saturated fat intake could substantially decrease population-wide cholesterol and blood pressure levels and consequently reduce CHD events. Our aim was to explore the potential reduction in CHD mortality 